\title{
Case Report \\ Yersinia pseudotuberculosis Infection Complicated with Bacteremia in a 10-Month-Old Boy
}

\author{
Takuro Kamura ${ }^{(D)},{ }^{1}$ Yuhei Tanaka $\left(\mathbb{D},{ }^{1,2}\right.$ Naoya Tsumura $\left(\mathbb{D},{ }^{1}\right.$ Takashi Ohya ${ }^{(D)}{ }^{1}$ \\ and Yuki Okamatsu $\mathbb{D}^{1}$ \\ ${ }^{1}$ Department of Pediatrics, Aso Iizuka Hospital, 3-83 Yoshio Town, Iizuka 820-8505, Japan \\ ${ }^{2}$ Department of Pediatrics and Child Health, Kurume University School of Medicine, 67 Asahi Town, Kurume 830-0011, Japan
}

Correspondence should be addressed to Yuhei Tanaka; yuhei.tuk.first@gmail.com

Received 13 June 2020; Revised 28 October 2020; Accepted 10 November 2020; Published 28 November 2020

Academic Editor: Nur Arslan

Copyright (c) 2020 Takuro Kamura et al. This is an open access article distributed under the Creative Commons Attribution License, which permits unrestricted use, distribution, and reproduction in any medium, provided the original work is properly cited.

\begin{abstract}
Yersinia pseudotuberculosis (Y. pseudotuberculosis) infection complicated with bacteremia rarely occurs. Y. pseudotuberculosis infection is also known to produce various symptoms similar to Kawasaki disease (KD) due to the production of Y. pseudotuberculosis-derived mitogen (YPM), an exotoxin with superantigen activity. Moreover, it causes terminal ileitis and is responsible for appendix swelling. Here, we report a case of Y. pseudotuberculosis infection in a 10-month-old boy who was brought to our hospital due to fever, watery stool, and poor vitality. Abdominal echocardiography revealed wall thickening of the entire colon and appendix swelling; therefore, he was admitted and treated with antibiotics for bacterial enteritis or appendicitis. After the antibiotic administration, facial skin rashes and hand and foot edema developed. However, he had 5/6 major symptoms of KD and was diagnosed with Y. pseudotuberculosis infection because of its presence in the blood and stool cultures. Thereafter, antibacterial therapy improved his symptoms and increased the inflammatory response. After his hospital discharge, the skin on his fingers showed desquamation like that of KD. Y. pseudotuberculosis infection should be considered as a differential disease in $\mathrm{KD}$, terminal ileitis, and appendicitis. Furthermore, its infection route and culture methods should also be carefully considered.
\end{abstract}

\section{Introduction}

Yersinia species are anaerobic Gram-negative bacilli belonging to the Enterobacteriaceae family, which develop bacterial enteritis by ingesting contaminated mountain or well water. Among them, Yersinia pseudotuberculosis (Y. pseudotuberculosis) is known to cause various symptoms in children, including Kawasaki disease (KD). Herein, we report a case of an infant diagnosed with enteritis and bacteremia due to Y. pseudotuberculosis based on blood and stool culture results.

\section{Case Presentation}

Written informed consent was obtained from the guardian of the patient for publication of this case report and accompanying images.
A 10-month-old boy was brought to our hospital with fever, watery stool, and poor vitality. He developed fever from the $1^{\text {st }}$ day, water stool (15-20 times a day) from the $2^{\text {nd }}$ day, and poor vitality from the $7^{\text {th }}$ day of illness. Vomiting occurred twice only on the $2^{\text {nd }}$ day. He was hospitalized on the $7^{\text {th }}$ day due to persistent symptoms and poor oral intake and vitality. He was previously healthy, with no recent history of travel, contact with sick individuals, or exposure to pets. His body temperature was $40.8^{\circ} \mathrm{C}$; pulse, $184 \mathrm{bpm}$ (normal); blood pressure, 121/66 $\mathrm{mmHg}$; and respiratory rate, 36 breathes/min with an oxygen saturation of $99 \%$ on room air. On physical examination, he was nonresponsive and had cervical lymphadenopathy, increased bowel sounds, and peripheral cold sensation. No other lymph node swelling, rash, or edema was observed (Figure 1). Initial laboratory results revealed elevated inflammatory markers (white blood cell (WBC) count, 15,750/ $\mu \mathrm{L}$; C-reactive 


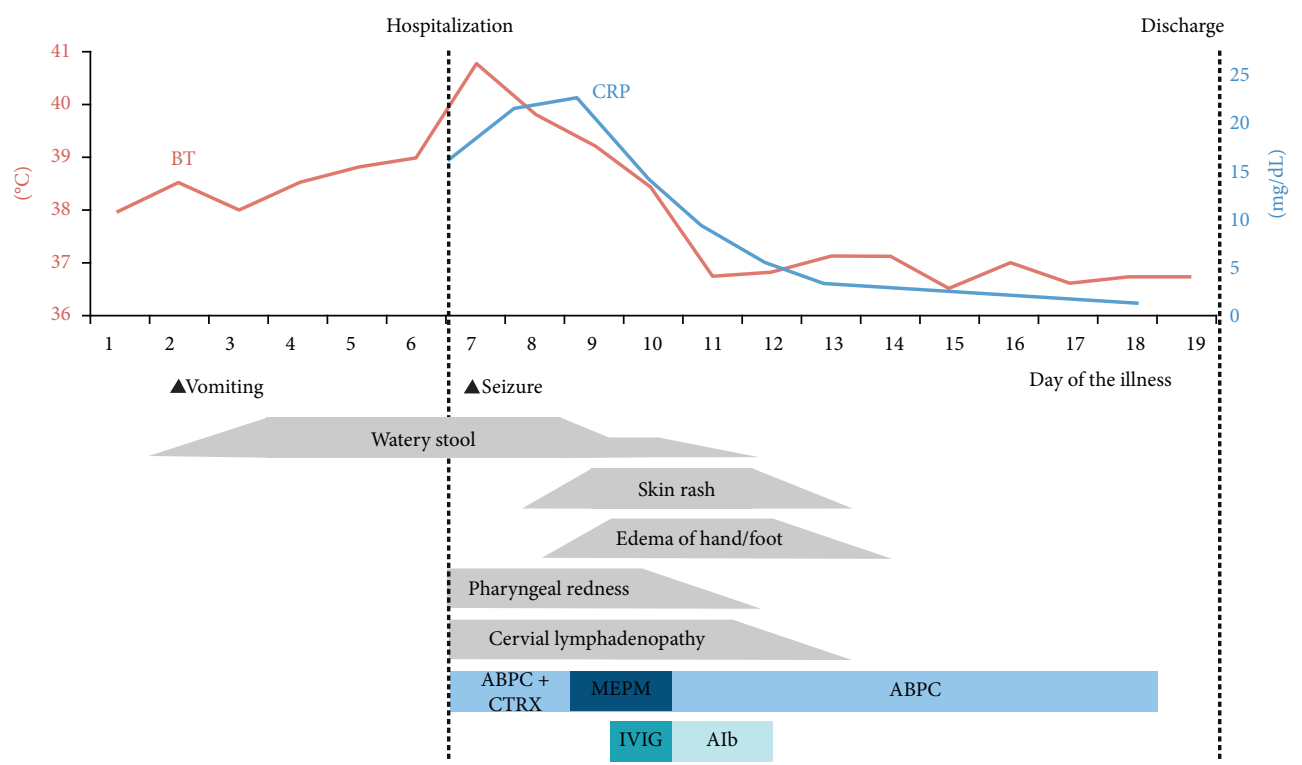

FIGURE 1: Clinical course of the patient. BT, body temperature; CRP, C-reactive protein; ABPC, ampicillin; CTRX, ceftriaxone; MEPM, meropenem: IVIG, intravenous immunoglobulin; Alb, albumin.

protein (CRP), $16.83 \mathrm{mg} / \mathrm{dL}$; and procalcitonin, $8.4 \mathrm{ng} / \mathrm{mL}$ ) and hyponatremia $(130 \mathrm{mmol} / \mathrm{L})$. Cervical ultrasonography revealed mild reactive lymphadenopathy (Figure 2). Abdominal ultrasonography (Figure 3) and computed tomography (CT) (Figure 4) revealed pitting-like wall thickness of the entire colon and an enlarged appendix.

Initially, estimating the pathogenic bacteria and infection route was difficult through interviews; therefore, a broad-spectrum antibiotic therapy was administered (ampicillin: ABPC, $200 \mathrm{mg} / \mathrm{kg} / \mathrm{day}+$ ceftriaxone (CTRX), $100 \mathrm{mg} / \mathrm{kg} / \mathrm{day})$. On the same day, 2-3-min convulsions occurred with upturn of the eyeball and poor complexion. Convulsions were identified as systemic tonic, without leftright differences. Cranial CT revealed no abnormal findings such as cerebral edema or neoplastic lesions, and cerebrospinal fluid examination revealed normal leukocyte levels or hypoglycemia (cerebrospinal fluid (CSF): glucose level of $73 \mathrm{mg} / \mathrm{dL}$ as compared to simultaneous blood glucose level of $113 \mathrm{mg} / \mathrm{dL}$, protein of $13 \mathrm{mg} / \mathrm{dL}$, and cell count of 4 cells/ $\mu \mathrm{L}$ with $58 \%$ cells being neutrophil).

On day 9 of illness, symmetrical pale erythema without blisters and bulges were observed on the patient's face and trunk. Follow-up laboratory results on the same day were as follows: WBC, $13290 / \mu \mathrm{L}$ and CRP, $23.3 \mathrm{mg} / \mathrm{dL}$. The blood culture on the $7^{\text {th }}$ day of the illness (hospitalization date) was reported to be positive for Gram-negative rod and was identified as Y. pseudotuberculosis. On the next day, the guardian was reinterviewed, revealing that they had been using spring water from nearby valleys for domestic purposes. The spring water was boiled and then used to melt the formula (artificial milk). Antibiotics were changed to meropenem (MEPM, $120 \mathrm{mg} / \mathrm{kg} /$ day), and intravenous immunoglobulin (IVIG, $500 \mathrm{mg} / \mathrm{kg} /$ day) was administered for hypogammaglobulinemia $(443 \mathrm{mg} / \mathrm{dL})$. On day 10 of illness, the fever subsided. On day 11, antibiotics were changed to ABPC (200 mg/kg/day) based on the antibiotic sensitivity to $Y$. pseudotuberculosis. Intravenous albumin $(10 \mathrm{~g} /$ dose/day) was also administered because of body weight gain and edema associated with hypoalbuminemia. These treatments gradually improved his watery stool, rashes, and cervical lymphadenopathy. Oral intake became possible on day 12 of illness, antibiotics were continuously infused until day 18, and he was discharged on day 19. During the hospitalization, no symptoms of acute renal failure were suspected. Finally, Y. pseudotuberculosis was detected from the stool culture and loop-mediated isothermal amplification (LAMP), and Y. pseudotuberculosisderived mitogen (YPM) antibody was also increased, resulting in the diagnosis of enteritis and bacteremia due to $Y$. pseudotuberculosis. After discharge, he presented desquamation of both hands on the $30^{\text {th }}$ day of illness. He has been under follow-up care without coronary artery lesions.

\section{Discussion}

Yersiniosis is a zoonotic infection occurring in domestic and wild animals; humans are considered incidental host that do not contribute to the natural disease cycle. The genus Yersinia includes 18 species, three of which are important human pathogens: Yersinia pestis, Yersinia enterocolitica, and Y. pseudotuberculosis. Yersinia infections are widespread globally, but most frequently reported in Europe [1]. It was first reported in Japan in 1972, and since then, sporadic cases and outbreaks of childhood diarrhea have been reported. Yersinia enterocolitica infections are common in eastern, whereas $Y$. pseudotuberculosis infections are common in the western region in Japan, and are closely related to the carriage of wild animals in each region [2]. Transmission of yersiniosis is largely food-borne and occasionally water-borne (untreated landslides and well water). Yersinia is isolated from animals such as pigs, dogs, cats, rabbits, and monkeys, as well as from rodents such as rats 


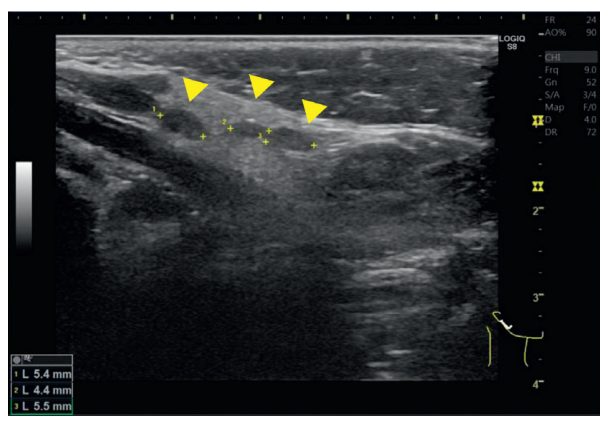

(a)

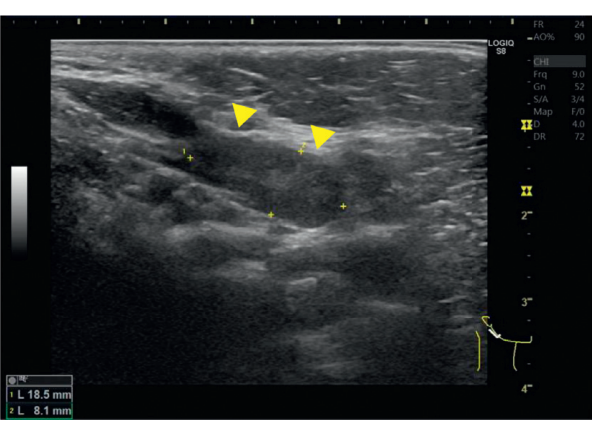

(b)

FIgURE 2: Cervical ultrasonography revealing mild reactive lymphadenopathy.

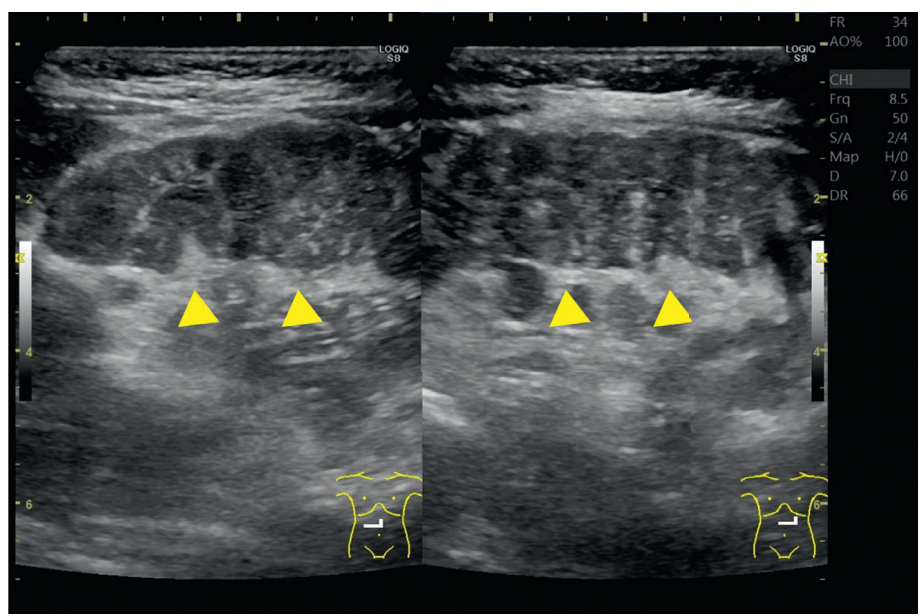

(a)

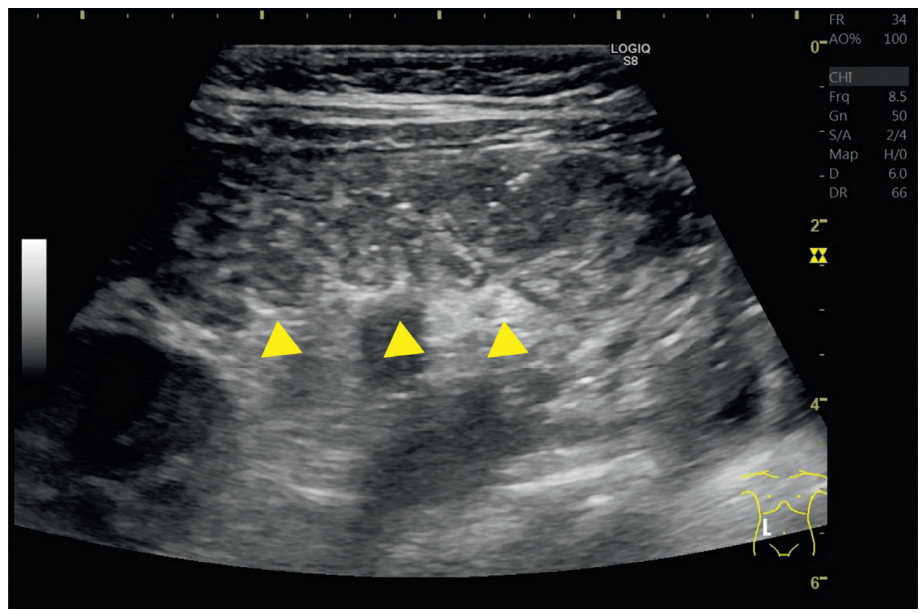

(b)

FIGURE 3: Abdominal ultrasonography revealing intraperitoneal lymphadenopathy, wall thickness of the entire colon.

and raccoons that contaminate the water surface. In addition, as it is widely distributed in wildlife, meat and raw milk contaminated with feces also cause infections. Its incubation period is $2-20$ (average, 8 ) days, and infants may become sick even with small doses.

Acute yersiniosis presenting with right lower abdominal pain, fever, vomiting, leukocytosis, and mild diarrhea [3] may be misinterpreted as acute appendicitis. In typical cases, abdominal ultrasonography and CT reveal thickening of the terminal ileum and enlarged lymph nodes around the intestinal tract [4]. In this case, similar imaging finding and appendix swelling were also revealed; thus, inflammatory bowel disease and acute appendicitis were suspected as differential diagnosis. The prevalence of acute appendicitis in young children is low, i.e., 1-6/10000 in children from birth to 4 years, but $19-28 / 10000$ in those aged $4-14$ years [5]. 

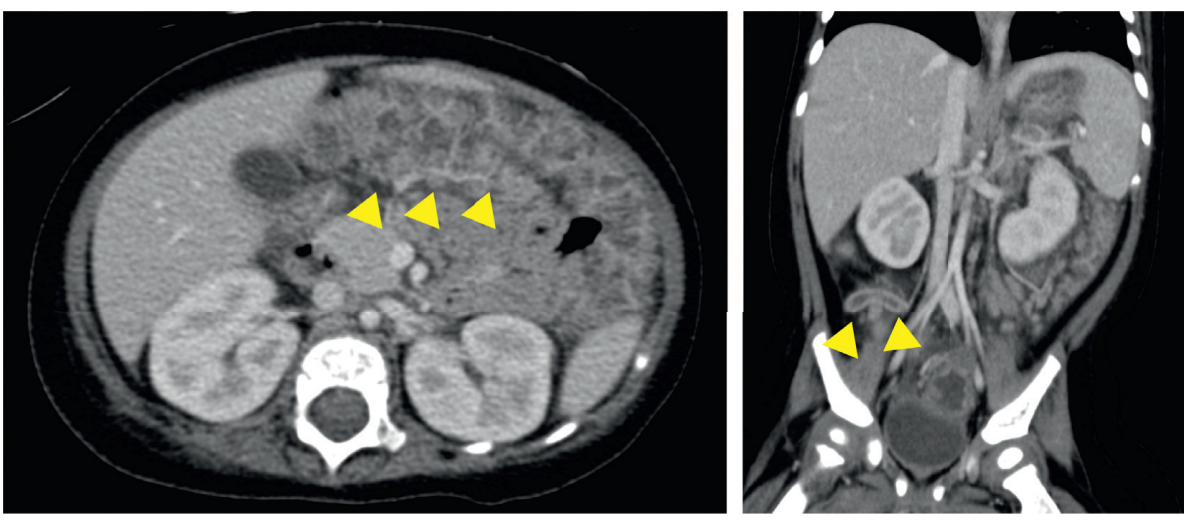

(a)
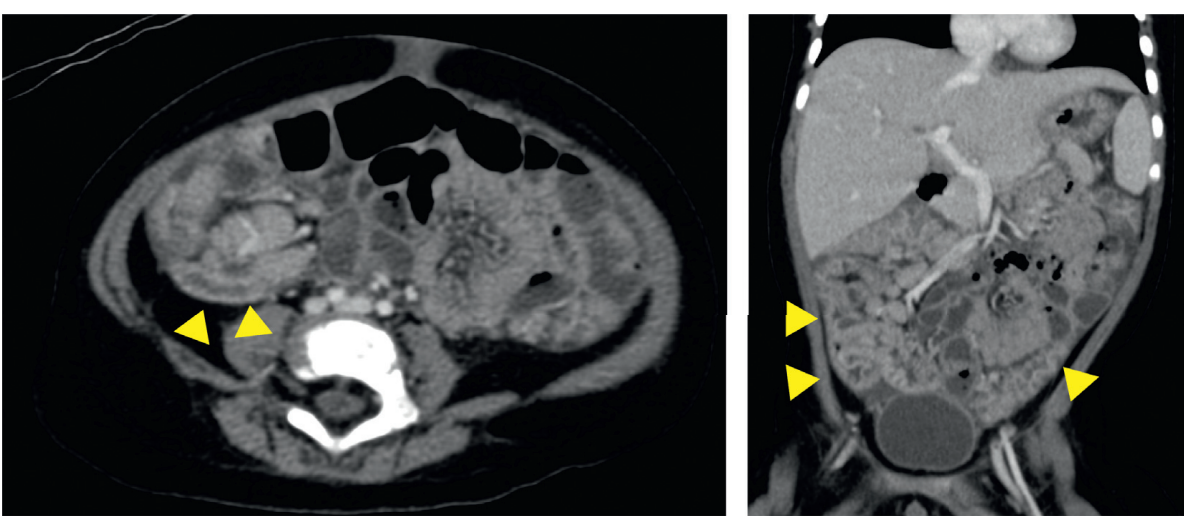

(b)

FIgURE 4: Abdominal computed tomography revealing pitting-like wall thickness of the entire colon, mesenteric lymphadenopathy of the right lower abdomen, and enlarged appendix.

Yersiniosis should be considered as a differential disease when diagnosing terminal ileitis and appendicitis in young children, especially up to 4 years old.

Furthermore, Y. pseudotuberculosis infection has been known to manifest various symptoms including those of $\mathrm{KD}$, i.e., $13 \%$ of patients have been reported to have met the diagnostic criteria of KD [6]. In addition, $10 \%$ of patients with KD have also been reported to test positive for anti-YPT or anti-YPM antibodies, and the positive group had a higher incidence of coronary sequelae (18\% in the positive group vs. $1 \%$ in the negative group) [7]. Moreover, among patients with $Y$. pseudotuberculosis infection, frequencies of diarrhea and abdominal pain in the family and use of natural water sources were reported to be significantly higher in the Y. pseudotuberculosis antibody-positive group [8]. In $\mathrm{KD}$, especially those with gastrointestinal symptoms, Y. pseudotuberculosis infection should be suspected, and therefore, culture and antibody titration should be performed. Moreover, even in patients diagnosed with Y. pseudotuberculosis infection, IVIG should be administered when antibacterial treatment is ineffective and meets the diagnostic criteria for $\mathrm{KD}$, and coronary artery evaluation with echocardiography should also be performed regularly.

Yersinia is generally difficult to detect in culture. For efficient detection, a low temperature $\left(25^{\circ} \mathrm{C}-29^{\circ} \mathrm{C}\right)$ should be used with a special selective medium, for example, efsulodin- irgasan-novobiocin (CIN) agar or CHROMagar Yersinia enterocolitica (CAYe) [9]. Therefore, when submitting stool culture, "Yersinia" should be clearly indicated as the target bacterium. Nevertheless, some case reports demonstrated difficulty in identifying Yersinia, resulting in not achieving a definitive diagnosis. In this case, the diagnosis of yersiniosis was possible from culture results; however, it should be noted that even if the culture is negative, serum antibody titer measurement or LAMP-based assay can be used to diagnose yersiniosis [10].

This case was complicated with bacteremia due to Y. pseudotuberculosis. Although similar cases have been reported, most of them are adult patients with underlying disorders, such as diabetes mellitus, liver cirrhosis, iron overload, malignant tumor, and after organ transplantation [11-14]. Very few cases of bacteremia due to $Y$. pseudotuberculosis have been reported in children without risk factors [14]. Y. pseudotuberculosis migrates to the mesenteric lymph node across Peyer's patch in the small intestine after ingestion [15]. Considering the findings of total colon thickness and mesenteric lymphadenopathy, $Y$. pseudotuberculosis possibly migrated further systemically from the mesenteric lymph nodes to the blood system.

Furthermore, symptoms did not improve despite of the initial antibiotic therapy. Convulsions even occurred for several minutes on the $7^{\text {th }}$ day of illness. Based on the CSF examination results, encephalitis was considered to be 
unlikely the cause of convulsions. Although acute encephalopathy has been reported to occur as a rare complication of $Y$. pseudotuberculosis infection [16, 17], mild encephalopathy cannot be denied because head MRI examination was not performed. However, postconvulsive consciousness was clear, without recurrent convulsions and prolongation of consciousness disorder; thereby, the patient is suspected to have simple febrile seizure only. Antibiotic therapy is effective for sepsis, but its effectiveness for other clinical symptoms is unknown. Antibiotics therapy was considered ineffective against YPM, which has a superantigen activity, and causes various types of systemic damage [18]. Subsequently, early antibiotic therapy finally succeeded, although the mortality rate of bacteremia due to Y. pseudotuberculosis was reported to be up to $75 \%$ [12].

In addition, his guardian stated in an interview during the hospitalization that they do not take natural water, but further interview revealed that "spring water from a nearby valley" had been used to melt the patient's formula (artificial milk).

Y. pseudotuberculosis infection rarely occurs and should be considered as an important differential disease in KD or appendicitis. The route of infection and culture methods should also be carefully considered, and serum antibody titers should be measured if $Y$. pseudotuberculosis infection is suspected.

\section{Conflicts of Interest}

The authors declare no conflicts of interest regarding the publication of this paper.

\section{Acknowledgments}

Special thanks are due to Jun Abe of National Center for Child Health and Development, who measured YPM, and Kazunobu Ouchi and Ippei Miyata of Kawasaki Medical University, who performed the Yersinia pseudotuberculosis LAMP method.

\section{References}

[1] European Centre for Disease Prevention and Control, Yersiniosis-Annual Epidemiological Report 2018, https://www. ecdc.europa.eu/en/publications-data/yersiniosis-annualepidemiological-report-2018, 2018.

[2] H. Fukushima, "Epidemiology of pathogenic Yersinia and their detection method," Japanese Journal of Food Microbiology, vol. 28, no. 2, pp. 104-113, 2011.

[3] American Academy of Pediatrics, Edited by D. W. Kimberlin, Ed., "Yersinia enterocolitica and Yersinia pseudotuberculosis infections," in Red Book: 2018-2021 Report of the Committee on Infection Diseases, M. T. Brady, M. A. Jackson, and S. S. Long, Eds., pp. 891-895, American Academy of Pediatrics, Itasca, IL, USA, 31th edition, 2018.

[4] T. Matsumoto, M. Iida, T. Sakai, Y. Kimura, and M. Fujishima, "Yersinia terminal ileitis: sonographic findings in eight patients," American Journal of Roentgenology, vol. 156, no. 5, pp. 965-967, 1991.

[5] Elsevier Saunders, "Acute Appendicitis," in Nelson Textbook of Pediatrics, R. M. Kliegman, B. Stanton, J. S. Geme, and
N. Schor, Eds., pp. 1887-1894, Elsevier Saunders, Philadelphia, USA, 20th edition, 2016.

[6] N. Takeda, "Various symptoms and complications of Yersinia infection," The Journal of Pediatric Infectious Disease and Immunology, vol. 29, no. 1, pp. 67-72, 2017.

[7] T. Horinouchi, K. Nozu, K. Hamahira et al., "Yersinia pseudotuberculosis infection in Kawasaki disease and its clinical characteristics," BMC Pediatrics, vol. 15, no. 177, 2015.

[8] H. Nakajima, H. Kariya, and T. Kishimoto, "A serological survey of Yersinia pseudotuberculosis infection (1999 2016)," The Journal of the Japanese Association for Infectious Diseases, vol. 92, no. 3, pp. 353-357, 2018.

[9] N. K. Thuan, K. Naher, R. Kubo, T. Taniguchi, and H. Hayashidani, "Evaluation of chromogenic medium for selective isolation of Yersinia," Food Hygiene and Safety Science, vol. 57, no. 5, pp. 166-169, 2016.

[10] S. Toyoda, D. Hirano, T. Makino et al., "Case of a 12 year-old with Yersinia pseudotuberculosis infection meeting the diagnostic criteria of the Kawasaki disease," Japanese Journal of Pediatrics, vol. 69, no. 11, pp. 1819-1823, 2016.

[11] W. K. Rathmell, P. Arguin, S. Chan, and A. Yu, "Yersinia pseudotuberculosis bacteremia and splenic abscess in a patient with non-insulin-dependent diabetes mellitus," The Western Journal of Medicine, vol. 170, no. 2, pp. 110-112, 1999.

[12] M. V. Zonneveld, J. M. Droogh, M. W. J. A. Fieren, I. C. Gyssens, T. V. Gelder, and W. Weimar, "Yersinia pseudotuberculosis bacteremia in a kidney transplant patient," Nephrology Dialysis Transplantation, vol. 17, no. 12, pp. 2252-2254, 2002.

[13] A. Renvoise, N. Lemaitre, G. Saintenoy et al., "Spontaneous ascitic fluid infection and bacteremia due to Yersinia pseudotuberculosis in a liver transplant patient," International Journal of Infectious Diseases, vol. 34, pp. 122-125, 2015.

[14] A. J. Kaasch, J. Dinter, T. Goeser, G. Plum, and H. Seifert, "Yersinia pseudotuberculosis bloodstream infection and septic arthritis: case report and review of the literature," Infection, vol. 40, no. 2, pp. 185-190, 2012.

[15] A. Marra and R. R. Isberg, "Invasin-dependent and invasinindependent pathways for translocation of Yersinia pseudotuberculosis across the Peyer's patch intestinal epithelium," Infection and Immunity, vol. 65, no. 8, pp. 3412-3421, 1997.

[16] T. Niizuma, K. Obinata, N. Matsunaga, A. Kamata, and K. Kinoshita, "Sibling cases of the Yersinia pseudotuberculosis infection: the younger brother developed acute encephalopathy, renal failure and the older sister presented Kawasaki disease like symptoms," The Journal of Pediatric Infectious Disease and Immunology, vol. 22, no. 3, pp. 211-216, 2016.

[17] H. Kaito, K. Kamei, M. Ogura et al., "Acute encephalopathy and tubulointerstitial nephritis associated with Yersinia pseudotuberculosis," Pediatric International, vol. 54, no. 6, pp. 926-928, 2012.

[18] J. Abe, M. Onimaru, S. Matsumoto et al., "Clinical role for a superantigen in Yersinia pseudotuberculosis infection," Journal of Clinical Investigation, vol. 99, no. 9, pp. 1823-1830, 1997. 